\title{
Macrophage polarization and its role in the pathogenesis of acute lung injury/acute respiratory distress syndrome
}

\author{
Xuxin Chen ${ }^{1} \cdot$ Jian Tang ${ }^{1} \cdot$ Weizheng Shuai ${ }^{2}$ Jiguang Meng ${ }^{1} \cdot$ Jian Feng $^{3} \cdot$ Zhihai Han $^{1}(\mathbb{C}$
}

Received: 30 May 2020 / Revised: 30 May 2020 / Accepted: 6 July 2020 / Published online: 10 July 2020

(c) Springer Nature Switzerland AG 2020

\begin{abstract}
Purpose Macrophages are highly plastic cells. Under different stimuli, macrophages can be polarized into several different subsets. Two main macrophage subsets have been suggested: classically activated or inflammatory (M1) macrophages and alternatively activated or anti-inflammatory (M2) macrophages. Macrophage polarization is governed by a highly complex set of regulatory networks. Many recent studies have shown that macrophages are key orchestrators in the pathogenesis of acute lung injury (ALI)/acute respiratory distress syndrome (ARDS) and that regulation of macrophage polarization may improve the prognosis of ALI/ARDS. A further understanding of the mechanisms of macrophage polarization is expected to be helpful in the development of novel therapeutic targets to treat ALI/ARDS. Therefore, we performed a literature review to summarize the regulatory mechanisms of macrophage polarization and its role in the pathogenesis of ALI/ARDS.

Methods A computer-based online search was performed using the PubMed database and Web of Science database for published articles concerning macrophages, macrophage polarization, and ALI/ARDS.

Results In this review, we discuss the origin, polarization, and polarization regulation of macrophages as well as the role of macrophage polarization in various stages of ARDS. According to the current literature, regulating the polarized state of macrophages might be a potential therapeutic strategy against ALI/ARDS.
\end{abstract}

Keywords Acute lung injury $\cdot$ Acute respiratory distress syndrome $\cdot$ Macrophage $\cdot$ Macrophage polarization

\section{Introduction}

Acute lung injury (ALI) and acute respiratory distress syndrome (ARDS) are common and devastating clinical disorders characterized by increased non-hydrostatic

Responsible Editor: John Di Battista.

Xuxin Chen, Jian Tang and Weizheng Shuai Co-first authors.

Jian Feng

fg5001@foxmail.com

$\bowtie$ Zhihai Han

zhihaihandoctor@163.com

1 Department of Pulmonary and Critical Care Medicine, The Sixth Medical Center of Chinese, PLA General Hospital, No. 6 Fucheng Road, Beijing 100037, China

2 Department of ICU, The Sixth Medical Center of Chinese, PLA General Hospital, Beijing 100037, China

3 Department of Cardiology, The Affiliated Hospital of Southwest Medical University, No. 25 Taiping Street, Luzhou 646000, China extravascular lung water, reduced pulmonary compliance, and acute hypoxemic respiratory failure [1]. Despite substantial progress in mechanical ventilatory strategies, significant advances in the quality of care of critically ill patients, and an enhanced understanding of molecular mechanisms, the mortality rate among patients with ALI/ARDS remains unacceptably high $[2,3]$.

The pathophysiological mechanism of ALI/ARDS is believed to be associated with numerous target and effector cells [1]. Among these cells, lung macrophages are the most important cells of the innate immune system and form the first line of defense against airborne particles and microbes $[4,5]$. Macrophages recognize pathogen-associated molecular patterns and trigger innate immunity and host defenses [6]. Macrophages also reportedly participate in the entire pathogenesis of ALI/ARDS, including modulation of inflammatory responses and repair of damaged lung tissues $[4,7$, 8].

Macrophages are highly plastic cells that display versatile functional phenotypes depending on microenvironmental stimuli [9]. Macrophages commonly exist in two distinct 
subsets: the classically activated (M1) macrophages or the alternatively activated (M2) macrophages [10]. M1/M2 macrophages have different functions and transcriptional profiles [9]. Moreover, the balance of the M1/M2 phenotype governs the fate of an organ in inflammation or injury [11]. In the acute exudative phase of ALI/ARDS, lung macrophages are M1 polarized. Continuous M1 polarization can release tumor necrosis factor $\alpha$ (TNF- $\alpha$ ), interleukin 1 (IL-1), nitric oxide (NO), and reactive oxygen species (ROS) to induce a severe inflammatory response [11]. In contrast, excessive M2 polarization contributes to a pathological fibroproliferative response and pulmonary fibrosis in the later fibroproliferative phase of ALI/ARDS [12, 13].

The findings of the present review are organized into three sections. First, the origin of macrophages are presented. Second, the polarization phenotypes and regulation of macrophages are discussed. Third, the role of macrophage polarization in ALI/ARDS is reviewed. Notably, this review is meant to be a broad overview for researchers seeking to understand the role of macrophage polarization in the development and prognosis of ALI/ARDS. The goal of the present review is to further our understanding of the physiological and pathological mechanisms that modulate macrophage polarization in ALI/ARDS.

\section{Origin of macrophages}

Macrophages have long been thought to be derived from bone marrow hematopoietic stem cells (HSCs) [14, 15]. In response to instructive cytokines and growth factors, bone marrow HSCs produce several discrete intermediate progenitor populations including common myeloid progenitors, granulocyte and macrophage progenitors, macrophage and dendritic cell progenitors, and common monocyte progenitors, which increasingly lose self-renewing capacity as they commit to the mononuclear phagocyte lineage $[16,17]$. Mature HSC-derived monocytes then enter the blood circulation and splenic reservoir [17]. When associated mobilization signaling is activated, circulating monocytes penetrate through the capillary endothelial cell layer, migrate into many different organs, and switch to tissue-resident macrophages [16].

However, recent studies have shown that a significant number of tissue macrophages are maintained independent of blood monocytes and are prenatally derived from primitive macrophages existing within the yolk sac or fetal liver (e.g., brain microglia, liver Kupffer cells, cardiac macrophages, and other macrophages that reside in the lungs, spleen, bone marrow, and peritoneal cavity) $[18,19]$. In the steady state, these macrophages renew locally, exhibit selfmaintenance, and carry out a variety of clearance and organspecific trophic functions [14, 20,21]. Under inflammation, immune dysfunction, and pathologic conditions, the number of macrophages often increases dramatically [22]. Tissueresident macrophage homeostasis can be destroyed by local amplification of resident macrophages and the recruitment of circulating monocytes $[14,17]$. Therefore, tissue-resident macrophages have a dual origin and consist of variably mixed populations of resident macrophages of embryonic origin and bone marrow-derived blood monocytes depending on the host status [19]

\section{Polarization phenotype and polarization regulation}

\section{Polarization phenotype}

Macrophage polarization is a process whereby macrophages phenotypically mount a specific phenotype and functional response to different pathophysiological conditions and surrounding microenvironments [10, 23]. In general, two main macrophage phenotypes have been suggested: classically activated or inflammatory (M1) macrophages and alternatively activated or anti-inflammatory (M2) macrophages $[10,11]$. However, M1/M2 polarization does not completely reflect the phenotypic populations of macrophages. The origin and properties of some other macrophage subsets remain unclear, but these subsets are known to be involved as the main players in some human pathologies; these subsets include tumor-associated macrophages (TAMs), CD169 ${ }^{+}$ macrophages, and $\mathrm{T}$ cell receptor-positive macrophages [24].

M1 macrophages are typically polarized by Th1-related cytokines such as interferon $\gamma$ (IFN- $\gamma$ ) and TNF- $\alpha$ as well as by microbicidal stimuli such as lipopolysaccharide (LPS) recognition. These macrophages produce higher levels of TNF- $\alpha$, IL-1, IL-6, IL-12, chemokine CCL8, IL-23, monocyte chemotactic protein 1 (MCP-1), macrophage inflammatory protein 2 (MIP-2), ROS, and cyclooxygenase 2 (COX-2), and they strongly express inducible NO synthase (iNOS), CD16, and CD32. Functionally, this population of macrophages is mainly engaged in proinflammatory, chemotaxis, radical formation, matrix degradation, antimicrobial, and antitumoral activities [25, 26]. Conversely, M2 macrophages are generated in response to Th2 cytokines such as IL-4 and IL-13 as well as anti-inflammatory cytokines such as IL-10 and transforming growth factor $\beta$ (TGF- $\beta$ ) [27]. These macrophages have an anti-inflammatory cytokine profile characterized by low production of IL-12 and high production of IL-1 receptor antagonist, chemokine CCL18, arginase 1 (Arg-1), found in inflammatory zone 1 (Fizz1), chitinase 3-like 3 (Chi313), IL-10, and TGF- $\beta$. Functionally, M2 macrophages suppress effector T cells, promote tissue remodeling, and facilitate tumor development; however, they are beneficial for inflammation resolution, parasite 
clearance, tolerogenesis, scavenging, angiogenesis, and tissue repair [27, 28].

\section{Polarization regulation}

Accumulating evidence indicates that identifying the polarized states of macrophages and switching the macrophage polarization from M1 to M2 or vice versa (repolarization or reprograming) could be novel diagnostic or therapeutic strategies for a wide variety of autoimmune and inflammatory diseases $[8,9,11,25,29]$. However, the precise regulatory mechanisms of macrophage polarization remain incompletely understood. Macrophage polarization is a rigorously controlled process involving a set of signaling pathways and transcriptional and post-transcriptional regulatory networks [23, 30, 31] (Fig. 1).

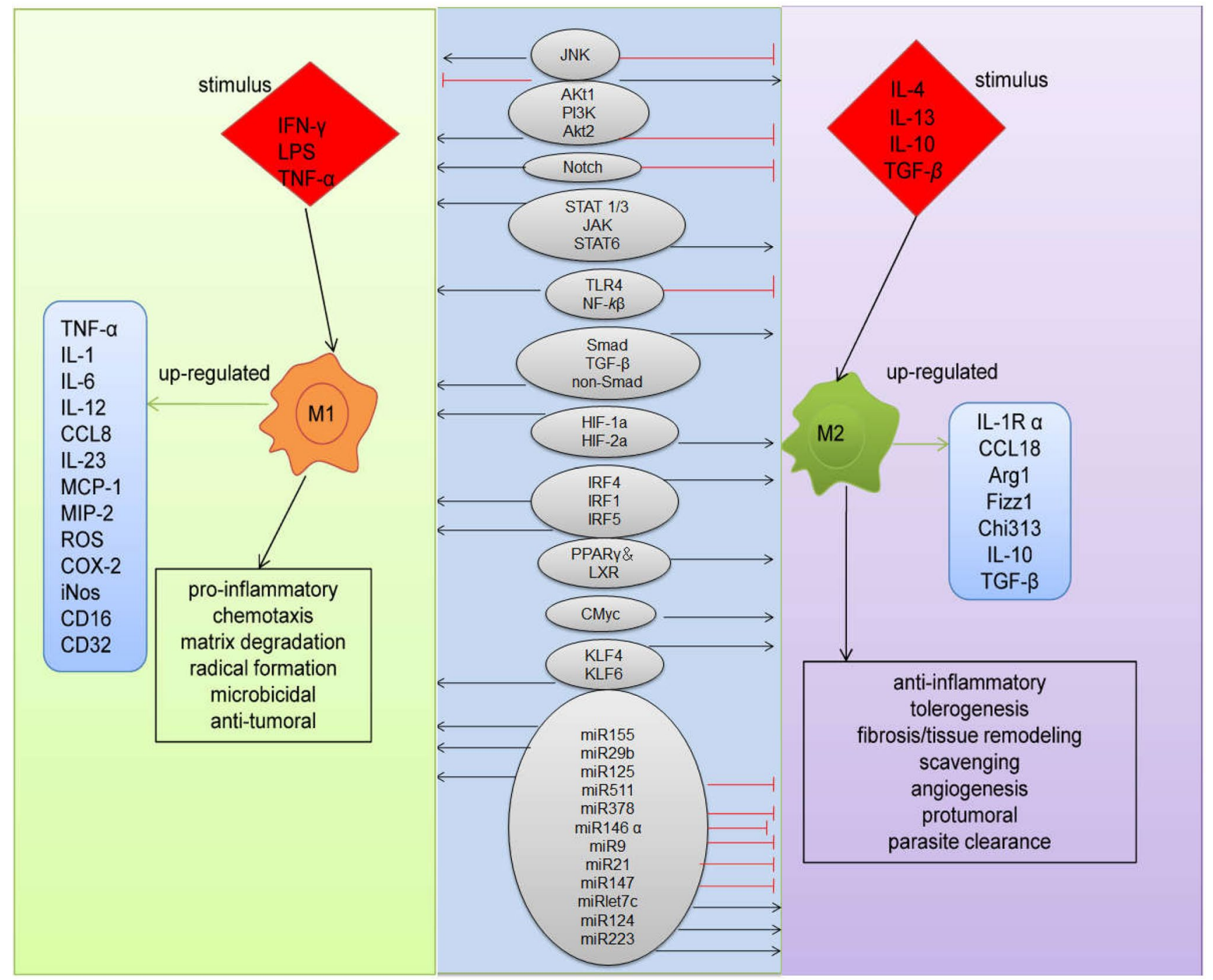

Fig. 1 Regulatory mechanisms and expression and functional characteristics of M1/M2 macrophages. M1 macrophage polarization is usually induced in vitro by IFN- $\gamma$, TNF- $\alpha$, and/or LPS stimulation. M2-type activation is usually modeled in vitro by Th2 cytokines (IL-4 and IL-13), IL-10, and TGF- $\beta$ stimulation. The main regulatory pathways, transcription factors, and miRs of M1/M2 macrophage polarization are outlined. The main genes and functions that are characteristic of either the M1 or M2 polarized state are also demonstrated. See text for further details. IFN- $\gamma$ interferon $\gamma, T N F-\alpha$ tumor necrosis factor $\alpha, I L$ interleukin, $M C P-1$ monocyte chemotactic protein $1, M I P-2$ macrophage inflammatory protein $2, R O S$ reactive oxy- gen species, $C O X-2$ cyclooxygenase 2 , iNOS inducible nitric oxide synthase, $L P S$ lipopolysaccharide, $T G F-\beta$ transforming growth factor $\beta$, Arg-1 arginase 1, Fizzl found in inflammatory zone 1, Chi313 chitinase 3-like 3, JNK Jun N-terminal kinase, PI3K phosphoinositide 3-kinase, STAT signal transducer and activator of transcription, JAK Janus family kinase, TLR toll-like receptor, $N F-\kappa B$ nuclear transcription factor- $\kappa \mathrm{B}, H I F$ hypoxia-inducible transcription factor, IRF interferon regulatory factor, $P P A R$ peroxisome proliferator-activated receptor, $L X R$ liver $\mathrm{X}$ receptor, $K L F$ Krüppel-like factor, $m i R$ microRNA 


\section{JNK signaling pathway}

The c-Jun N-terminal kinase (JNK) signaling pathway belongs to the superfamily of mitogen-activated protein kinases (MAPKs) [29]. Three distinct isoforms in the JNK family have been identified: JNK1, JNK2, and JNK3 [29]. JNK is reportedly required for M1-related inflammation and fibrosis, insulin resistance, macrophage infiltration, M1 macrophage polarization, and the M1-associated gene expression profile [32-36]. In previous studies, downregulation of phosphor-JNK induced macrophage polarization toward the M2 subset in rat white adipose tissue macrophages and in the murine macrophage cell line RAW264.7 [37, 38]. However, in RAW264.7 modified with malignant fibrous histiocytomaamplified sequence 1 and praja ring finger 2, blockade of the JNK pathway did not influence the expression of M2 macrophage polarization biomarkers [39]. In contrast, Hao et al. [40] reported that high expression of JNK is involved in IL-4-induced M2 macrophage polarization and that JNK increases the expression of downstream transcription c-Myc and M2 markers. The regulatory effect of JNK on macrophage polarization might be mediated by the cyclic adenosine monophosphate/protein kinase A pathway [38].

\section{PI3K/Akt signaling pathway}

Phosphoinositide 3-kinases (PI3Ks), a class of lipid kinases, are constitutively expressed in various immune cells [41]. Members of the PI3K family are divided into four classes based on their structural features and substrate specificity: $\mathrm{I}_{\mathrm{A}}, \mathrm{I}_{\mathrm{B}}$, II, and III [42]. Class $\mathrm{I}_{\mathrm{A}}$ PI3Ks can convert phosphatidylinositol 4,5-bisphosphate ( $\left.\mathrm{PIP}_{2}\right)$ into the lipid second messenger phosphatidylinositol $(3,4,5)$-trisphosphate $\left(\mathrm{PIP}_{3}\right)$, which results in the activation of a downstream transduction signal [41]. Akt (also known as protein kinase B) is a family of three serine/threonine protein kinases (Akt1, Akt2, and Akt3) and is the most prominent effector of PI3K [43]. Once the conversion of $\mathrm{PIP}_{2}$ to $\mathrm{PIP}_{3}$ occurs, the downstream proteins PDK1 and Akt will be activated [41, 44]. Binding of PDK1 and Akt to PIP ${ }_{3}$ further leads to the phosphorylation of Akt [42]. Increasing numbers of studies are suggesting that the PI3K/Akt signaling pathway also plays crucial roles in macrophage activation and gene expression [29, 45]. Zhang et al. [46] reported that inhibition of the PI3K/Akt pathway can depress M2 macrophage polarization. In fact, the PI3K/Akt signaling pathway differentially contributes to macrophage polarization depending on the Akt isoform [43]. Studies have shown that $\mathrm{Akt}^{-/-}$macrophages express high levels of iNOS, TNF- $\alpha$, and IL- 6 , while $\mathrm{Akt}^{-/-}$macrophages express high levels of Fizz1, Chi313, and IL-10 [43]. These data suggest that Akt1 ablation gives rise to an M1 phenotype and that Akt2 ablation results in an M2 phenotype [47]. Additionally, microRNA (miR)-155 and its target CAAT/enhancer-binding protein $\beta$ (C/EBP $\beta)$ are critical for Akt isoform-dependent M1/M2 polarization of macrophages [43]. Akt1 leads to upregulation of miR-155 and consequent inhibition of $\mathrm{C} / \mathrm{EBP} \beta$, which finally blunts the M1 macrophage reaction [43, 47]. Conversely, Akt2 promotes M1 phenotype transformation by an opposite influence on miR-155 and C/EBP $\beta$ [43].

\section{Notch signaling pathway}

The Notch signaling pathway is an evolutionarily conserved pathway [48]. The Notch receptor family is divided into four classes: Notch1, Notch2, Notch3, and Notch4. These classes, especially Notch1, are widely expressed in various organs and tissues [49]. Ligands of Notch receptors are divided into two families: Delta-like 1, 3, and 4 and the JAG-type ligands Jagged-1 and -2 [50].The Notch signaling pathway plays a critical role in M1 phenotype polarization [51]. Activation of Notch signaling promotes macrophage polarization from M2 to M1 and increases M1 macrophage-associated gene expression. In contrast, macrophages deficient in canonical Notch signaling typically show the M2 phenotype [51, 52]. Notch signaling can not only promote M1 macrophage differentiation, but also prevent M2 macrophage differentiation by binding with Delta-like 4, thus modulating the expression of M2-specific gene expression and apoptotic cell death [53]. Notch signaling can function through both canonical and non-canonical pathways [54]. In Notch canonical signaling, the binding of a Notch ligand with its receptor triggers cleavage of Notch by a furin-like convertase [29]. Receptors release the Notch intracellular domain (NICD) by two subsequent proteolytic cuts, the first by a disintegrin and metalloproteinase domain (ADAM)-type proteinase and the second by a $\gamma$-secretase, which results in the liberation and nuclear translocation of NICD [54]. The binding of NICD to the sequence-specific DNA-binding factor recombinant binding protein for immunoglobulin region $\mathrm{KJ}$ (RBP-J) leads to the formation of a transcriptional activator complex that induces the transcriptional expression of genes under the control of Notch [29]. Activated Notch1 and expression of the Notch target genes significantly modulate the expression of TNF- $\alpha$, IL-6, and IL-10 through activation of nuclear transcription factor- $\kappa \mathrm{B}(\mathrm{NF}-\kappa \mathrm{B})$ [51]. However, in the noncanonical pathway, NICD activity is independent of both RBP-J and cleavage of the $\gamma$-secretase complex [55].

\section{JAK/STAT signaling pathway}

The Janus family kinases (JAKs), including JAK1, JAK2, JAK3, and TYK2, belong to the subgroup of non-receptor protein tyrosine kinases [56]. JAKs exert their effect mainly through activation of cytosolic DNA-binding proteins called signal transducers and activators of transcription (STATs). 
STATs include seven family members (STATs $1-6$, including two STAT5 genes) [57]. Accumulating evidence has confirmed that many cytokines regulate macrophage phenotypes and activation via the JAK/STAT signaling pathway [57-59]. STAT1 is the most important mediator of M1 macrophage polarization induced by IFN- $\gamma$ [31]. Binding of the IFN- $\gamma$ ligand to its receptor induces JAK1/2-mediated tyrosine phosphorylation and subsequent dimerization of STAT1, which binds as a homodimer to cis elements known as IFN- $\gamma$-activated sites in the promoter of target M1 signature genes [31]. Hence, the IFN- $\gamma / \mathrm{JAK} / \mathrm{STAT} 1$ pathway is believed to be a key component of M1 phenotype modulatory factors [29]. Oh et al. [60] found that asaronic acid could attenuate macrophage activation toward the M1 phenotype through inhibition of JAK/STAT1 signaling. Liang et al. [61] reported that downregulation of suppressor of cytokine signaling 1 (SOCS1) could promote M1 macrophage polarization via the JAK1/STAT1 pathway. However, the role of type I IFN-mediated signaling in macrophage polarization remains unclear [29]. Type I IFN can exhibit anti-inflammatory activity in certain conditions, and IFN $\alpha / \beta$ receptor signaling inhibits resistance to Listeria monocytogenes infection by inducing macrophage apoptosis [29]. This suggests that IFN $\gamma$ - and IFN $\alpha / \beta$-mediated STAT 1 activation might have a different effect on macrophage polarization in vivo [31]. IFN- $\gamma$ activates JAK/STAT-1 phosphorylation, which leads to M1-like macrophage polarization. In contrast, IFN- $\alpha / \beta$ induces the expression of SOCS3, which inhibits STAT-1 phosphorylation. Moreover, STAT3 is reportedly an important transcription factor for the activation of macrophages and augmentation of inflammation [62]. Suppressing STAT3 activity has a protective effect on LPS-induced ALI via inhibition of expression of proinflammatory genes and polarization of M1 macrophages [62]. STAT6 is a crucial transcription factor in the activation of M2 macrophages induced by IL-4 or IL-13 [63]. IL-4 type I and II receptors activate STAT6, which in turn promotes expression of genes typical of M2 polarization [63]. SOCS proteins are described as feedback inhibitors of the JAK/STAT signaling pathway [64]. In the process of macrophage polarization, JAK/STAT signaling is activated while the expression of SOCS1 and SOCS3 is upregulated [64]. This upregulation of SOCS1 and SOCS3 in turn suppresses the action of STAT1 and STAT3, respectively, which can terminate innate and adaptive immune responses [64, 65]. Moreover, the recognition component of the classical complement cascade $\mathrm{C} 1 \mathrm{q}$ might also inhibit STAT1 phosphorylation with subsequent suppression of M1 macrophage polarization [29].

\section{TLR4/NF-KB signaling pathway}

TLR engagement, particularly TLR4 stimulated by LPS and other microbial ligands, leads to NF- $\mathrm{KB}$ activation and production of inflammatory mediators associated with the M1 phenotype [63]. TLR4 signaling includes both the myeloid differentiation factor 88 (MyD88)-dependent and MyD88-independent pathways [4]. In the MyD88-independent pathway, TLR4 recruits TIR domain-containing adaptorinducing IFN- $\beta$ and TIR domain-containing adaptor molecule 2 [4]. Activation of the MyD88-indpendent pathway leads to the activation and translocation of IFN regulatory factor 3 (IRF3) in the nucleus, thus inducing the secretion of type I IFN [4]. IRF3 is involved in regulating M1 polarization and M1-associated gene induction [23]. IFN-stimulated genes include chemokine CXCL9 and CXCL10, which are characteristic of classical M1 macrophage activation [23]. In the MyD88-dependent pathway, TLR4 recruits MyD88, MyD88 adaptor-like, IL-1 receptor-associated kinase, and TNF receptor-associated factor 6 [66]. The activation of this pathway finally induces the activation and translocation of $\mathrm{NF}-\kappa \mathrm{B}$ in the nucleus [66]. NF- $\kappa \mathrm{B}$ regulates the expression of a large number of inflammatory genes including TNF- $\alpha$, IL- $1 \beta$, cyclooxygenase 2 , and IL-6, which are associated with macrophage M1 activation [23]. However, NF- $\mathrm{kB}$ activation also activates a genetic program essential for resolution of inflammation and M2 polarization of TAMs [63]. Otherwise, macrophage polarization is actually related to the expression ratio of various TLRs on macrophages [23]. The TLR4/TLR2 ratio is significantly higher in M1 than M2 macrophages [23].

\section{TGF- $\beta$ signaling pathway}

The secretory TGF- $\beta$ protein family includes TGF- $\beta 1$, TGF- $\beta 2$, TGF- $\beta 3$, activins, and growth factors, which have pleiotropic effects on adaptive immunity [67]. The TGF- $\beta$ receptor complex is composed of two type I TGF- $\beta$ receptors (TGF- $\beta$ RI) and two type II TGF- $\beta$ receptors (TGF$\beta$ RII) [67]. TGF- $\beta$ triggers signaling in cells by binding to the TGF- $\beta$ receptor complex [68]. Active TGF- $\beta$ binds to a dimer of TGF- $\beta$ RII, which associates with a dimer of TGF- $\beta R I$ to form a tetrameric receptor complex. TGF- $\beta$ RII then phosphorylates the cytoplasmic domain of TGF- $\beta$ RI [68], allowing TGF- $\beta$ RI to directly phosphorylate $\operatorname{Smad} 2 / 3$. Once phosphorylated, Smad $2 / 3$ forms a complex with either Smad4 or TIF $1 \gamma$ to modulate gene expression by binding to Smad-responsive regulatory regions [67]. This signal transduction is called the Smad-dependent signaling pathway. TGF- $\beta$ can also trigger non-Smad-mediated or Smad-independent signaling events (e.g., via MAPK, Rho GTPases, or PI3K) to regulate gene expression [67]. In Smad-dependent pathways, TGF- $\beta$ upregulates the expression of M2 phenotype genes and induces macrophages to undergo M2 polarization [69]. Conversely, in Smad-independent signaling pathways, TGF- $\beta$ activates MAPK pathways, JNK, $\mathrm{p} 38$, and NF- $\kappa$ B by the TGF- $\beta$-activated kinase 1 protein, which can 
reprogram macrophages to the M1 phenotype, particularly when the Smad-dependent signaling pathway is blocked [69]. Moreover, the effect of Smad-dependent pathways on M2 polarization is under the control of Smad7. Smad7 can bind to TGF- $\beta R I$ and prevent Smad2/3/4 complex formation, thereby inhibiting the production of anti-inflammatory M2 cytokines [69]. Additionally, TGF- $\beta$ reportedly induces an M2-like phenotype via SNAIL upregulation and blockade of TGF- $\beta /$ SNAIL signaling, thus restoring the production of proinflammatory cytokines [70].

\section{HIF-a}

Hypoxic conditions can directly influence macrophage polarization [23]. Hypoxia-inducible transcription factor (HIF) is an important regulator of hypoxia-related macrophage polarization [69]. HIF is composed of an HIF- $\beta$ subunit and HIF- $\alpha$ subunit [69]. HIF- $\beta$ is constitutively expressed in the cell, whereas HIF- $\alpha$ is an oxygen-sensitive subunit [69]. Hypoxia exerts its effect on macrophage polarization via two isoforms of HIF- $\alpha$ : HIF- $1 \alpha$ and HIF-2 $\alpha$ [71]. In a hypoxic microenvironment, the activity of the prolyl4-hydroxylase domain enzyme decreases and HIF- $\alpha$ consequently accumulates, which promotes the formation of an HIF- $\alpha /$ HIF- $\beta$ dimer [69]. Takeda et al. [72] reported that HIF- $1 \alpha$ and HIF- $2 \alpha$ might drive macrophage polarization by modulating NO homeostasis. In fact, under hypoxia, either HIF- $1 \alpha$ or HIF- $2 \alpha$ is activated depending on the extent of the hypoxia [69]. HIF-1 $\alpha$ induces iNOS synthesis and M1 phenotype formation, but HIF- $2 \alpha$ activates Arg- 1 and induces M2 polarization [69].

\section{IRF}

IRFs were originally described as regulators of type I IFN expression and signaling and are important mediators of macrophage polarization [31]. There are nine IRFs in mammals, and several members have been reported in relation to a specific phenotype [30, 31]. IRF4 was shown to regulate M2 macrophage polarization and its associated gene expression profile $[73,74]$, and this process is likely associated with histone demethylase JMJD3 [75]. High expression of IRF5 was observed in M1 macrophages and was subsequently described as an important regulator in M1 macrophage polarization [30]. IRF5 can be directly recruited to gene promoters associated with the M1 phenotype and then activate the transcription of proinflammatory genes and inhibit anti-inflammatory IL-10 expression [31, 76]. IRF1 can be described as an important mediator in M1 polarization via cooperation with NF- $\mathrm{KB}$ and antagonization of IRF4 function [30].

\section{PPARY and LXRa}

Peroxisome proliferator-activated receptor $\gamma(\operatorname{PPAR} \gamma)$, a nuclear receptor, has been described as an important determinant of IL-4 or IL-13-induced M2 macrophage polarization [77]. PPAR $\gamma$ is constitutively expressed and is a master regulator of lipid metabolism in adipose tissue macrophages [31]. PPAR $\gamma$ expression can also be induced by IL- 4 and IL-13, indicating that M2 polarization in the context of Th2 cell responses might also involve PPAR $\gamma$ [78]. Studies have shown the role of PPAR $\gamma$ in promoting M2 activation to protect mice from insulin resistance by using PPAR $\gamma$-deficient macrophages [77]. Researchers have proposed that obesity and/or inflammatory stress can lead to a polarized macrophage phenotypic switch from M2 to M1 in adipose tissue, leading to further inflammation and insulin resistance in the absence of PPAR $\gamma$-mediated proinflammatory gene repression [31]. Several studies have shown that cross talk exists between PPAR $\gamma$ and the IL-4/STAT6 axis or endogenous PPAR $\gamma$ ligands, which might coordinately control the M2 phenotype [31, 79]. Another nuclear receptor, liver X receptor $\alpha(\mathrm{LXR} \alpha)$, is similar to PPAR $\gamma$ and plays an important role in M2 macrophage polarization. LXR $\alpha$ can upregulate Arg-1 expression and suppress inflammatory signaling in macrophages [30].

\section{c-Myc}

$\mathrm{c}-\mathrm{Myc}$ is a pleiotropic transcription factor involved in a variety of biological process, including cell proliferation, biosynthetic metabolism, and apoptosis [80]. In the immune compartment, c-Myc is involved in the process of M2 macrophage polarization [23]. The transcription factor c-Myc can be activated by IL-4 and different stimuli sustaining M2-like polarization, after which it translocates to the nucleus [80]. c-Myc not only directly promotes M2 polarization-associated gene expression, but also induces an M2-like phenotype via upregulation of STAT6 and PPAR $\gamma$ [80].

\section{KLF4 and KLF6}

Krüppel-like factors (KLFs) are a subfamily of the zinc finger class of DNA-binding transcriptional regulators [81]. Among the members of this gene family, KLF4 has been identified as an essential regulator of macrophage M1/M2 polarization and attendant functions [82]. In one study, KLF4 expression was robustly induced in M2 macrophages and strongly reduced in M1 macrophages [82]. Using myeloid-specific transcription factor KLF4 knockout mice, Liao et al. [82] demonstrated the role of KLF4 in regulating M2 polarization of macrophages and protecting mice from obesity-induced insulin resistance. KLF4 cooperated with STAT6 to induce an M2 genetic program and inhibit M1 
targets by suppressing NF- $\kappa \mathrm{B} / \mathrm{HIF}-1 \alpha$-dependent transcription [82]. Compared with KLF4, KLF6 has an adverse effect on macrophage M1/M2 polarization [83]. Date et al. [83] showed that KLF6 expression was enhanced by proinflammatory M1 stimuli and suppressed by M2 stimuli in human and murine macrophages. KLF6 promoted M1 polarization via cooperation with $\mathrm{NF}-\mathrm{\kappa B}$ and inhibited $\mathrm{M} 2$ polarization by suppressing PPAR $\gamma$ expression [83].

\section{miRs and polarization}

Functional miRs are believed to form a set of networks to modulate macrophage polarization and control the M1/M2 phenotype switch [69]. In response to macrophage reprogramming factor $(\mathrm{RF})$, a specific set of miR expression can be induced. RF-M1s induce the synthesis of miR-155, miR21, miR-29b, miR-125, miR-9, miR-146a, and miR-147, whereas RF-M2s induce the synthesis of miR-146b, miR511, miR-187, miR-378, miR-222, miR-27a, miR-125a-3p, and miR-125a-5p [69]. miR-155 reportedly plays an important role in Akt-regulated and IL-13-induced M1/M2 polarization [23, 84]. Overexpression or depletion of miR-155 promotes macrophages to exhibit the M1 or M2 phenotype by regulation of transcription factor C/EBP- $\beta$ [23]. miR155 increases stability of the TNF- $\alpha$ transcript, decreases synthesis of SOCS1, and decreases the effect of TGF- $\beta$ on M2 reprogramming [69]. miR-155 can also shift the M2 phenotype of TAM to M1, thereby promoting the development of anti-tumor immunity [85]. Similarly, miR-29b and miR-125 promote formation of the M1 phenotype, whereas miR-146a, miR-9, miR-21, and miR-147 have a negative effect on M1 polarization [69]. Ponomarev et al. [86] found that miR-124 regulates macrophage plasticity and deactivate macrophages in a $\mathrm{C} / \mathrm{EBP} \alpha$-dependent manner. Moreover, miR-223 can reportedly regulate macrophage polarization by targeting Pknox 1 [87]. Both miR-124 and miR-223 are believed to be associated with the immunosuppressive macrophage phenotype [23]. Banerjee et al. [88] found overexpression of miR-let-7c in M2-type macrophages and not in M1-type macrophages. Upregulation of miR-let-7c promotes M2 polarization [88]. In contrast, miR-511 and miR-378 are negative regulators of the macrophage M2 response [69].

\section{Role of macrophage polarization in ALI/ARDS}

ARDS is a devastating disease with distinct pathological stages, including an exudative phase, rehabilitation phase, and fibrotic phase $[8,25]$. Macrophages are highly plastic cells that play diverse roles in all three different pathological stages based on the microenvironment (Fig. 2). Two main classes of macrophages exist in the lung. The first class is alveolar macrophages (AMs), which reside within the lumen of the alveolus and are the most abundant population [89].
The main function of AMs is to phagocytize foreign particles and catabolize surfactant $[89,90]$. The other class is interstitial macrophages, which account for $30-40 \%$ of lung macrophages [89]. The function of interstitial macrophages is thought to be associated with tissue remodeling and repair, antigen presentation, and modulation of dendritic cell functions [89, 91]. AMs can be further divided into two subpopulations according to their functional state and sources: longlived resident AMs and recruited AMs [8]. The long-lived resident AMs act as a uniform, quiescent, and immunosuppressive population and mainly present the M2 phenotype $[8,92]$. However, peripheral blood monocytes are recruited into the alveolar lumen in response to certain stimuli [8]. This population of AMs are called recruited AMs and differentiate into macrophages with the M1 phenotype [93]. Accumulating evidence suggests that macrophages, including both resident AMs and recruited AMs, are key factors in the pathogenesis of ALI/ARDS [8].

\section{Exudative phase of ALI/ARDS}

In this stage, the resident AMs immediately shift to the predominant M1 phenotype in response to infection-induced activation of TLRs or other recognition receptors [94]. Excessive production of macrophages can contribute to tissue damage. These AMs release a variety of inflammatory cytokines such as IL- $1 \beta$, IL-6, MCP-1, MIP-2, TNF- $\alpha$, and ROS [95]. The neutrophils induced by these proinflammatory factors are then recruited from the intravascular space, cross the endothelium and epithelium, and finally move into the lungs and alveolar spaces. Excessive accumulation of proinflammatory factors and neutrophils can lead to lung tissue damage [8]. Recent studies have shown that some naturally derived or synthesized materials can ameliorate the prognosis of ALI in animal models by inhibiting M1 polarization of AMs [96-100]. Therefore, M1 macrophages serve as promoters in the process of lung tissue damage in ALI/ARDS. However, recent studies have demonstrated that M1 macrophages can protect against LPS-induced ALI and ventilator-induced lung injury via enhanced expression of amphiregulin, inhibiting the gene expression of proinflammatory cytokines and protecting the epithelial barrier [101].

\section{Rehabilitation phase of ALI/ARDS}

Following the exudative phase, the second phase of ALI/ ARDS is the rehabilitation phase. In the rehabilitation phase of ALI/ARDS, pathogenic factors are eliminated, and resident and recruited macrophages then shift from the M1 to the M2 phenotype [8]. The phagocytosis of apoptotic neutrophils by macrophages acts as one of the driving factors that promotes the M2 phenotype [102]. M2 macrophages can enhance the expression of IL-10, fibronectin 1, and the 


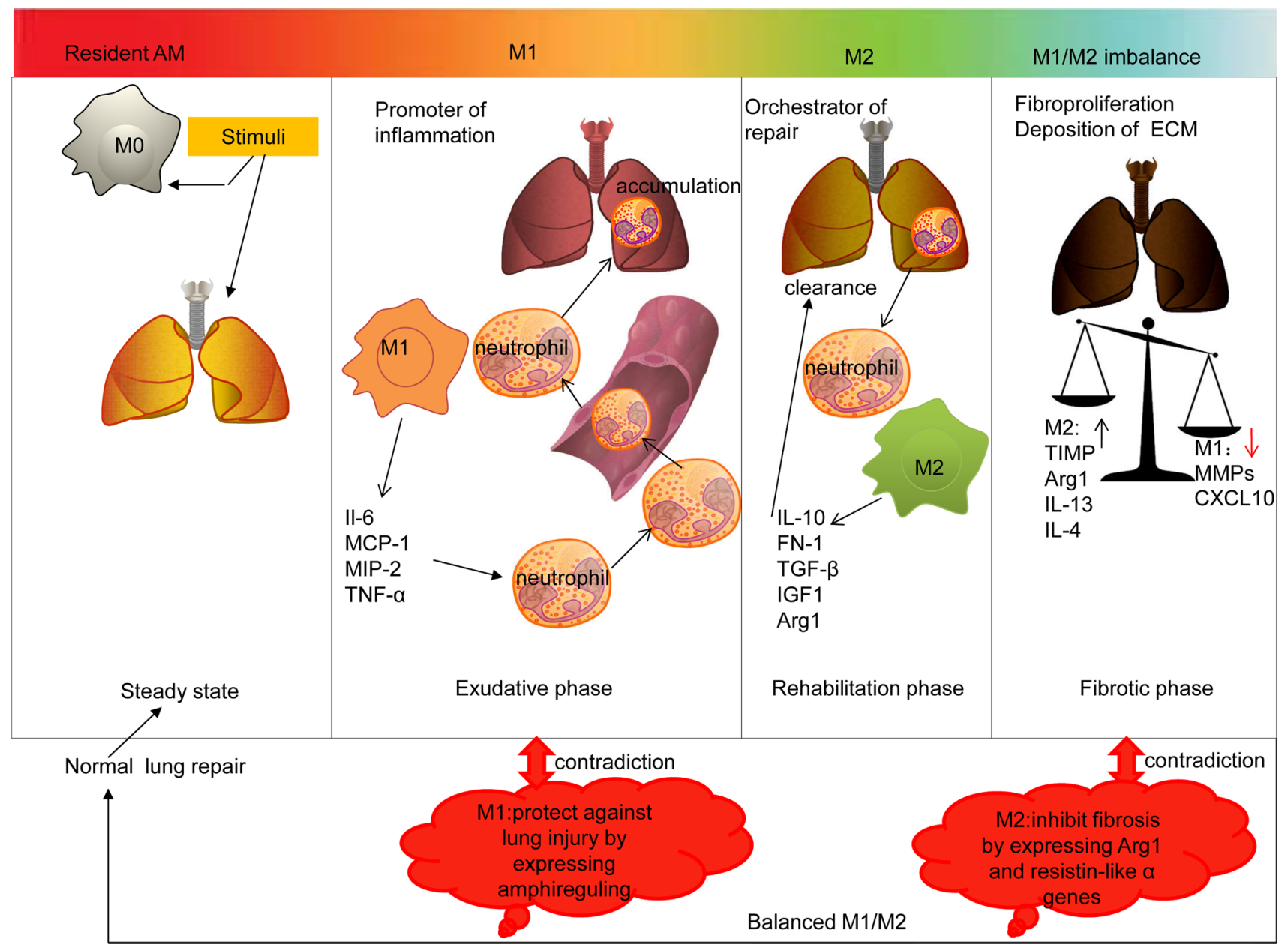

Fig. 2 Dynamics between M1 and M2 macrophages during ALI/ ARDS. Macrophages undergo dynamic changes in the different phases of ALI/ARDS, predominantly displaying the M1 or M2 phenotype. Under certain stimuli, normal resident AMs shift to the M1 phenotype during the exudative phase of ALI/ARDS. M1 macrophages release various proinflammatory cytokines at the site of inflammation, and neutrophils are then recruited from the circulation into the lungs and alveolar spaces. Excessive accumulation of proinflammatory factors and neutrophils promote progression of inflammation and lung injury. After causative factors are eliminated, macrophages shift from the M1 phenotype to the M2 phenotype, and ALI/ARDS progresses to the rehabilitation stage. M2 macrophages play an important role in inflammation resolution and lung tissue repair by limiting the levels of proinflammatory cytokines and enhancing the production of anti-inflammatory cytokines. Clear-

TGF- $\beta$-induced matrix-associated proteins BIG-H3 and insulin-like growth factor 1 and limit the levels of proinflammatory cytokines; this promotes repair of host tissues, reduces alveolar epithelial cell damage, and increases the pulmonary barrier function after inflammation $[8,103]$. Apart from the regulation of proinflammatory and antiinflammatory cytokines, M2 macrophages can also activate anti-inflammatory signaling, terminate proinflammatory responses, and finally promote rehabilitation of lung injury ance of apoptotic neutrophils and debris is also attributed to M2 macrophages, which are helpful for inflammation termination and lung tissue repair. During the later fibrotic phase of ALI/ARDS, the balance of M1/M2 macrophages determines the trend and extent of lung fibrosis. M1 macrophages release MMPs and CXCL10 to promote matrix degradation and decrease fibrosis. Persistence of M2 macrophages promotes fibroproliferation and ECM deposition by enhanced expression of TIMP, Arg-1, IL-13, and IL-4. Balanced M1/ M2 polarization shifts lung injury toward normal lung repair. $I L$ interleukin, $M C P-1$ monocyte chemotactic protein $1, M I P-2$ macrophage inflammatory protein $2, T N F-\alpha$ tumor necrosis factor $\alpha, R O S$ reactive oxygen species, $F N-1$ fibronectin $1, T G F-\beta$ transforming growth factor $\beta, I G F 1$, insulin-like growth factor 1, Arg- 1 arginase-1, TIMP tissue inhibitor of metalloproteinase, $M M P S$ matrix metalloproteinase, $E C M$ extracellular matrix

by the clearance of apoptotic neutrophils from inflammatory sites [8]. Phagocytosis of apoptotic neutrophils by M2 macrophages further increases the production of antiinflammatory factors such as IL-10 and TGF- $\beta$, which may be beneficial for inflammation control [103]. Phagocytosis also inhibits the expression of iNOS and stimulates the expression of Arg-1, thereby preventing ROS production [102]. In a Pseudomonas aeruginosa infection-induced ALI murine model, macrophages demonstrated peak expression 
of the M2 marker (transferrin receptor) during the resolution phases of lung injury in the alveolar space [102]. Moreover, Shirey et al. [104] reported that respiratory syncytial virus could induce AMs to produce IL-4 and IL-13, which contribute to M2 macrophage differentiation and disease resolution in respiratory syncytial virus-induced lung injury. Additionally, Tu et al. [105] found that methylprednisolone could attenuate LPS-induced ALI by increasing the number of M2 macrophages and induction of M2 polarization. These results suggest that M2 macrophages are key orchestrators in the regulation of lung damage and tissue repair in the rehabilitation phase of ALI/ARDS.

\section{Fibrotic phase of ALI/ARDS}

Pulmonary fibrosis, a late complication of ALI/ARDS, is characterized by fibroblast proliferation and excessive deposition of ECM $[8,106]$. The fibrotic phase of ALI/ARDS is linked to prolonged mechanical ventilation and increased mortality [106]. Following destruction of the basement membrane integrity, M1 and M2 macrophages are recruited into the lung tissue injury site to regulate the formation of fibrosis. M1 macrophages play an important role in matrix degradation by direct and indirect production of matrix metalloproteinases (MMPs) and a variety of antifibrotic cytokines such as CXCL10 $[107,108]$. The production of MMPs is important for remodeling of the ECM, which contributes to resolution of the pathological fibroproliferative response in the later phase of ALI/ARDS [107]. M1 macrophages can also induce myofibroblasts to express MMP13 and MMP-3 [107, 109]. In contrast to the expression of MMPs by M1 macrophages, M2 macrophages markedly express anti-inflammatory cytokines and tissue inhibitors of metalloproteinase, which impair remodeling of excessive ECM [107, 110]. Moreover, co-culture of M2 macrophages with myofibroblasts can induce complex ECM deposition [107]. Arg-1 is an important M2 macrophage-associated molecule that can degrade L-arginine into L-proline [27, 107]. It can be used to produce collagen by myofibroblasts [111]. Prolonged effects of IL-13 and IL-4 on AMs promote the presence of M2 macrophages and, finally, excessive fibrogenesis [8]. Therefore, M2 macrophages are usually believed to be more promotive of a fibroproliferative microenvironment. However, some recent contradictory results have been presented. IL-4-polarized M2 macrophages can reportedly inhibit fibrosis by expressing Arg-1 and resistinlike $\alpha$ genes (surface marker of the M2 phenotype) [112]. Wakayama et al. [113] found that dental pulp stem cells could attenuate bleomycin-induced lung fibrosis by inducing M2-like lung macrophages. Therefore, macrophages are paradoxically involved in the process of lung fibrosis. The magnitude of myofibroblast activation and deposition of ECM and the severity of lung tissue fibrosis may be partly dependent on the balance of M1 versus M2 macrophages in the local microenvironment of lung tissue injury.

\section{Summary and perspective}

This paper has reviewed the origin, polarization, and polarization regulation of macrophages and the role of macrophage polarization in various stages of ALI/ARDS. A much better understanding of the role that macrophage polarization plays in inflammatory, reparative, and fibrotic responses relevant to ALI/ARDS pathogenesis may pave the way for new therapeutic tools to effectively treat ALI/ARDS.

Macrophages are believed to have multiple origins, including HSCs, the yolk sac, and the fetal liver. A high degree of plasticity is the fundamental characteristic of the macrophage. There are two main polarization states of macrophages according to their responses to environmental stimuli: the M1 and M2 phenotypes. Macrophages can switch from one phenotype to another when the environmental stimuli change. Generally, M1 macrophages are mainly involved in proinflammatory activity and tissue damage, whereas M2 macrophages have converse properties. The regulation of macrophage polarization involves a highly complex set of regulatory networks at both the transcriptional and post-transcriptional levels.

Macrophages are important effector cells that participate in all stages of ALI/ARDS, including the exudative phase, rehabilitation phase, and fibrotic phase. In the exudative phase of ALI/ARDS, lung macrophages shift into the M1 phenotype and act as potent promoters to induce severe inflammatory responses and lung tissue damage. Following entry into the rehabilitation stage, M2 macrophages are increasingly prominent in lung tissues and serve as key orchestrators in regulating lung tissue damage and repair and promoting disease resolution. Excessive M2 polarization contributes to the pathological fibroproliferative response and pulmonary fibrosis in the later fibrotic phase of ALI/ ARDS. However, the functions of different macrophage subsets are not absolutely defined in each phase of ALI/ARDS. Some studies have shown that in the acute exudative phase of ALI/ARDS, M1 macrophages can attenuate the proinflammatory response and protect the epithelial barrier from injury. Similarly, the M2 phenotype is reportedly involved in the antifibrotic process in the fibrotic stage of ALI/ARDS. Therefore, the properties and functions of each macrophage subpopulation are not rigorously inflexible. Macrophages are paradoxically involved in inflammation, tissue repair, and fibrosis in ALI/ARDS. Nonetheless, targeting macrophage polarization for ALI/ARDS treatment has been proven effective and promising in many preclinical studies [96-99, 105, 112]. Limiting excessive proinflammatory responses and tissue damage and balancing excessive fibrosis and tissue repair via regulation of macrophage activation and 
polarization represent novel and potential therapeutic targets for ALI/ARDS. However, several issues remain unresolved, such as the underlying signaling pathways of macrophage polarization and the mechanisms of cell-cell communication in the process of macrophage activation and polarization. Thus, fully elucidating how macrophage polarization can be manipulated to improve the outcome of ALI/ARDS requires further time and effort. Although macrophage polarizationbased therapy for lung injury is still a long way off, we hope that this review provides valuable information that may be helpful in developing novel therapies for ALI/ARDS.

Acknowledgements The authors thank Angela Morben, DVM, ELS, from Liwen Bianji, Edanz Editing China (www.liwenbianji.cn/ac), for editing the English text of a draft of this manuscript.

Author contributions $\mathrm{XC}$ and JM contributed to paper revision and writing enhancement in grammar and language; JT and WS wrote the paper; $\mathrm{JF}$ and $\mathrm{ZH}$ are responsible for the idea and fund.

Funding The present study was co-supported by the National Natural Science Foundation of China (Grant \#81300050 and Grant \#31300946), the Beijing Natural Science Foundation (Grant \#7182163), and the Innovative Cultivation Foundation of The Sixth Medical Center of PLA General Hospital (Grant \#CXPY201902)

\section{Compliance with ethical standards}

Conflict of interest The authors declare that they have no conflict of interest.

Ethics approval This is a review. The local Ethics Committee of PLA General Hospital has confirmed that no ethical approval is required.

\section{References}

1. Nanchal RS, Truwit JD. Recent advances in understanding and treating acute respiratory distress syndrome. F1000Res. 2018;7(F1000 Faculty Rev):1322.

2. Chen X, Wu S, Tang L, Ma L, Wang F, Feng H, et al. Mesenchymal stem cells overexpressing heme oxygenase-1 ameliorate lipopolysaccharide-induced acute lung injury in rats. J Cell Physiol. 2019;234:7301-19.

3. Chen XX, Tang L, Han ZH, Wang WJ, Meng JG. Coculture with bone marrow-derived mesenchymal stem cells attenuates inflammation and apoptosis in lipopolysaccharide-stimulated alveolar epithelial cells via enhanced secretion of keratinocyte growth factor and angiopoietin-1 modulating the Toll-like receptor-4 signal pathway. Mol Med Rep. 2019;19:1891-902.

4. Chen XX, Tang L, Fu YM, Wang Y, Han ZH, Meng JG. Paralemmin-3 contributes to lipopolysaccharide-induced inflammatory response and is involved in lipopolysaccharide-Toll-like receptor-4 signaling in alveolar macrophages. Int J Mol Med. 2017;40:1921-31.

5. Gea-Sorlí S, Guillamat R, Serrano-Mollar A, Closa D. Activation of lung macrophage subpopulations in experimental acute pancreatitis. J Pathol. 2011;223:417-24.

6. Wu DD, Pan PH, Liu B, Su XL, Zhang LM, Tan HY, et al. Inhibition of alveolar macrophage pyroptosis reduces lipopolysaccharide-induced acute lung injury in mice. Chin Med J (Engl). 2015;128:2638-45.

7. Han J, Li C, Liu H, Fen D, Hu W, Liu Y, et al. Inhibition of lipopolysaccharide-mediated rat alveolar macrophage activation in vitro by antiflammin-1. Cell Biol Int. 2008;32:1108-15.

8. Huang X, Xiu H, Zhang S, Zhang G. The role of macrophages in the pathogenesis of ALI/ARDS. Mediators Inflamm. 2018;2018:1264913.

9. Shapouri-Moghaddam A, Mohammadian S, Vazini H, Taghadosi M, Esmaeili SA, Mardani F, et al. Macrophage plasticity, polarization, and function in health and disease. J Cell Physiol. 2018;233:6425-40.

10. Biswas SK, Chittezhath M, Shalova IN, Lim JY. Macrophage polarization and plasticity in health and disease. Immunol Res. 2012;53:11-24.

11. Patel U, Rajasingh S, Samanta S, Cao T, Dawn B, Rajasingh J. Macrophage polarization in response to epigenetic modifiers during infection and inflammation. Drug Discov Today. 2017;22:186-93.

12. Xiang J, Cheng S, Feng T, Wu Y, Xie W, Zhang M, et al. Neotuberostemonine attenuates bleomycin-induced pulmonary fibrosis by suppressing the recruitment and activation of macrophages. Int Immunopharmacol. 2016;36:158-64.

13. Janssen WJ, Barthel L, Muldrow A, Oberley-Deegan RE, Kearns MT, Jakubzick C, et al. Fas determines differential fates of resident and recruited macrophages during resolution of acute lung injury. Am J Respir Crit Care Med. 2011;184:547-60.

14. Ginhoux F, Guilliams M. Tissue-resident macrophage ontogeny and homeostasis. Immunity. 2016;44:439-49.

15. Davies LC, Taylor PR. Tissue-resident macrophages: then and now. Immunology. 2015;144:541-8.

16. Hettinger J, Richards DM, Hansson J, Barra MM, Joschko AC, Krijgsveld J, et al. Origin of monocytes and macrophages in a committed progenitor. Nat Immunol. 2013;14:821-30.

17. Pittet MJ, Nahrendorf M, Swirski FK. The journey from stem cell to macrophage. Ann N Y Acad Sci. 2014;1319:1-18.

18. Yona S, Kim KW, Wolf Y, Mildner A, Varol D, Breker M, et al. Fate mapping reveals origins and dynamics of monocytes and tissue macrophages under homeostasis. Immunity. 2013;38:79-91.

19. Davies LC, Jenkins SJ, Allen JE, Taylor PR. Tissue-resident macrophages. Nat Immunol. 2013;14:986-95.

20. Hashimoto D, Chow A, Noizat C, Teo P, Beasley MB, Leboeuf $\mathrm{M}$, et al. Tissue-resident macrophages self-maintain locally throughout adult life with minimal contribution from circulating monocytes. Immunity. 2013;38:792-804.

21. Sieweke MH, Allen JE. Beyond stem cells: self-renewal of differentiated macrophages. Science. 2013;342:1242974.

22. Gordon S, Plüddemann A. Tissue macrophages: heterogeneity and functions. BMC Biol. 2017;15:53.

23. Wang N, Liang H, Zen K. Molecular mechanisms that influence the macrophage $\mathrm{m} 1-\mathrm{m} 2$ polarization balance. Front Immunol. 2014;5:614.

24. Chávez-Galán L, Olleros ML, Vesin D, Garcia I. Much more than M1 and M2 macrophages, there are also CD169(+) and TCR(+) macrophages. Front Immunol. 2015;6:263.

25. Aggarwal NR, King LS, D'Alessio FR. Diverse macrophage populations mediate acute lung inflammation and resolution. Am J Physiol Lung Cell Mol Physiol. 2014;306:L709-L725725.

26. Fukui S, Iwamoto N, Takatani A, Igawa T, Shimizu T, Umeda M, et al. M1 and M2 monocytes in rheumatoid arthritis: a contribution of Imbalance of M1/M2 monocytes to osteoclastogenesis. Front Immunol. 2017;8:1958.

27. Gordon S, Martinez FO. Alternative activation of macrophages: mechanism and functions. Immunity. 2010;32:593-604. 
28. Zhang YH, He M, Wang Y, Liao AH. Modulators of the balance between M1 and M2 macrophages during pregnancy. Front Immunol. 2017;8:120.

29. Zhou D, Huang C, Lin Z, Zhan S, Kong L, Fang C, et al. Macrophage polarization and function with emphasis on the evolving roles of coordinated regulation of cellular signaling pathways. Cell Signal. 2014;26:192-7.

30. Hoeksema MA, Stöger JL, de Winther MP. Molecular pathways regulating macrophage polarization: implications for atherosclerosis. Curr Atheroscler Rep. 2012;14:254-63.

31. Lawrence T, Natoli G. Transcriptional regulation of macrophage polarization: enabling diversity with identity. Nat Rev Immunol. 2011;11:750-61.

32. Han MS, Jung DY, Morel C, Lakhani SA, Kim JK, Flavell RA, et al. JNK expression by macrophages promotes obesity-induced insulin resistance and inflammation. Science. 2013;339:218-22.

33. Yang $\mathrm{S}$, Wang $\mathrm{H}$, Yang $\mathrm{Y}$, Wang R, Wang $\mathrm{Y}$, Wu C, et al. Baicalein administered in the subacute phase ameliorates ischemiareperfusion-induced brain injury by reducing neuroinflammation and neuronal damage. Biomed Pharmacother. 2019;117:109102.

34. Shi Q, Zhao L, Xu C, Zhang L, Zhao H. High molecular weight hyaluronan suppresses macrophage M1 polarization and enhances IL-10 production in PM2.5-induced lung inflammation. Molecules. 2019;24:1766.

35. Yang J, Yang L, Tian L, Ji X, Yang L, Li L. Sphingosine 1-phosphate $(\mathrm{S} 1 \mathrm{P}) / \mathrm{S} 1 \mathrm{P}$ receptor $2 / 3$ axis promotes inflammatory M1 polarization of bone marrow-derived monocyte/macrophage via $\mathrm{G}(\alpha) \mathrm{i} / \mathrm{o} / \mathrm{PI} 3 \mathrm{~K} / \mathrm{JNK}$ pathway. Cell Physiol Biochem. 2018;49:1677-93.

36. Mao Y, Wang B, Xu X, Du W, Li W, Wang Y. Glycyrrhizic acid promotes M1 macrophage polarization in murine bone marrowderived macrophages associated with the activation of JNK and NF-кB. Mediators Inflamm. 2015;2015:372931.

37. Oliveira AG, Araujo TG, Carvalho BM, Guadagnini D, Rocha GZ, Bagarolli RA, et al. Acute exercise induces a phenotypic switch in adipose tissue macrophage polarization in diet-induced obese rats. Obes (Silver Spring). 2013;21:2545-56.

38. Wan S, Sun H. Glucagon-like peptide-1 modulates RAW264.7 macrophage polarization by interfering with the JNK/STAT3 signaling pathway. Exp Ther Med. 2019;17:3573-9.

39. Zhong J, Wang H, Chen W, Sun Z, Chen J, Xu Y, et al. Correction: Ubiquitylation of MFHAS1 by the ubiquitin ligase praja2 promotes M1 macrophage polarization by activating JNK and p38 pathways. Cell Death Dis. 2018;9:782.

40. Hao J, Hu Y, Li Y, Zhou Q, Lv X. Involvement of JNK signaling in IL4-induced M2 macrophage polarization. Exp Cell Res. 2017;357:155-62.

41. Ruse M, Knaus UG. New players in TLR-mediated innate immunity: PI3K and small Rho GTPases. Immunol Res. 2006;34:33-48.

42. Luyendyk JP, Schabbauer GA, Tencati M, Holscher T, Pawlinski R, Mackman N. Genetic analysis of the role of the PI3KAkt pathway in lipopolysaccharide-induced cytokine and tissue factor gene expression in monocytes/macrophages. J Immunol. 2008; 180:4218-26.

43. Arranz A, Doxaki C, Vergadi E, Martinez de la Torre Y, Vaporidi $\mathrm{K}$, Lagoudaki ED, et al. Akt1 and Akt2 protein kinases differentially contribute to macrophage polarization. Proc Natl Acad Sci USA. 2012;109:9517-22.

44. Fukao T, Koyasu S. PI3K and negative regulation of TLR signaling. Trends Immunol. 2003;24:358-63.

45. Bao L, Li X. MicroRNA-32 targeting PTEN enhances M2 macrophage polarization in the glioma microenvironment and further promotes the progression of glioma. Mol Cell Biochem. 2019;460:67-79
46. Zhang LL, Zhang LF, Shi YB. Down-regulated paxillin suppresses cell proliferation and invasion by inhibiting M2 macrophage polarization in colon cancer. Biol Chem. 2018;399:1285-95.

47. Xu F, Kang Y, Zhang H, Piao Z, Yin H, Diao R, et al. Akt1mediated regulation of macrophage polarization in a murine model of Staphylococcus aureus pulmonary infection. J Infect Dis. 2013;208:528-38.

48. Artavanis-Tsakonas S, Rand MD, Lake RJ. Notch signaling: cell fate control and signal integration in development. Science. 1999;284:770-6.

49. Espinoza I, Miele L. Notch inhibitors for cancer treatment. Pharmacol Ther. 2013;139:95-110.

50. Rutz S, Janke M, Kassner N, Hohnstein T, Krueger M, Scheffold A. Notch regulates IL-10 production by T helper 1 cells. Proc Natl Acad Sci U S A. 2008;105:3497-502.

51. Wei W, Li ZP, Bian ZX, Han QB. Astragalus Polysaccharide RAP induces macrophage phenotype polarization to M1 via the notch signaling pathway. Molecules. 2019;24:2016.

52. Zhang W, Xu W, Xiong S. Blockade of Notch1 signaling alleviates murine lupus via blunting macrophage activation and $\mathrm{M} 2 \mathrm{~b}$ polarization. J Immunol. 2010;184:6465-78.

53. Pagie S, Gérard N, Charreau B. Notch signaling triggered via the ligand DLL4 impedes M2 macrophage differentiation and promotes their apoptosis. Cell Commun Signal. 2018;16:4.

54. Vieceli Dalla Sega F, Fortini F, Aquila G, Campo G, Vaccarezza M, Rizzo P. Notch signaling regulates immune responses in atherosclerosis. Front Immunol. 2019;10:1130.

55. Ayaz F, Osborne BA. Non-canonical notch signaling in cancer and immunity. Front Oncol. 2014;4:345.

56. Ghoreschi K, Laurence A, O'Shea JJ. Janus kinases in immune cell signaling. Immunol Rev. 2009;228:273-87.

57. Murray PJ. The JAK-STAT signaling pathway: input and output integration. J Immunol. 2007;178:2623-9.

58. Hu X, Chen J, Wang L, Ivashkiv LB. Crosstalk among Jak-STAT, Toll-like receptor, and ITAM-dependent pathways in macrophage activation. J Leukoc Biol. 2007;82:237-43.

59. Hall CJ, Boyle RH, Astin JW, Flores MV, Oehlers SH, Sanderson LE, et al. Immunoresponsive gene 1 augments bactericidal activity of macrophage-lineage cells by regulating $\beta$-oxidationdependent mitochondrial ROS production. Cell Metab. 2013;18:265-78.

60. Oh H, Park SH, Kang MK, Kim YH, Lee EJ, Kim DY, et al. Asaronic acid attenuates macrophage activation toward M1 phenotype through inhibition of NF-KB pathway and JAK-STAT signaling in glucose-loaded murine macrophages. J Agric Food Chem. 2019;67:10069-78.

61. Liang YB, Tang H, Chen ZB, Zeng LJ, Wu JG, Yang W, et al. Downregulated SOCS1 expression activates the JAK1/STAT1 pathway and promotes polarization of macrophages into M1 type. Mol Med Rep. 2017;16:6405-11.

62. Zhao J, Yu H, Liu Y, Gibson SA, Yan Z, Xu X, et al. Protective effect of suppressing STAT3 activity in LPS-induced acute lung injury. Am J Physiol Lung Cell Mol Physiol. 2016;311:L868-L880880.

63. Sica A, Mantovani A. Macrophage plasticity and polarization: in vivo veritas. J Clin Invest. 2012;122:787-95.

64. Whyte CS, Bishop ET, Rückerl D, Gaspar-Pereira S, Barker RN, Allen JE, et al. Suppressor of cytokine signaling (SOCS)1 is a key determinant of differential macrophage activation and function. J Leukoc Biol. 2011;90:845-54.

65. Liu Y, Stewart KN, Bishop E, Marek CJ, Kluth DC, Rees AJ, et al. Unique expression of suppressor of cytokine signaling 3 is essential for classical macrophage activation in rodents in vitro and in vivo. J Immunol. 2008;180:6270-8. 
66. Molteni M, Gemma S, Rossetti C. The role of toll-like receptor 4 in infectious and noninfectious inflammation. Mediators Inflamm. 2016;2016:6978936.

67. Travis MA, Sheppard D. TGF- $\beta$ activation and function in immunity. Annu Rev Immunol. 2014;32:51-82.

68. Hata A, Chen YG. TGF- $\beta$ signaling from receptors to smads. Cold Spring Harb Perspect Biol. 2016;8:a022061.

69. Malyshev I, Malyshev Y. Current concept and update of the macrophage plasticity concept: intracellular mechanisms of reprogramming and $\mathrm{m} 3$ macrophage "switch" phenotype. Biomed Res Int. 2015;2015:341308.

70. Zhang F, Wang H, Wang X, Jiang G, Liu H, Zhang G, et al. TGF- $\beta$ induces M2-like macrophage polarization via SNAILmediated suppression of a pro-inflammatory phenotype. Oncotarget. 2016;7:52294-30606.

71. Imtiyaz HZ, Simon MC. Hypoxia-inducible factors as essential regulators of inflammation. Curr Top Microbiol Immunol. 2010;345:105-20.

72. Takeda N, O'Dea EL, Doedens A, Kim JW, Weidemann A, Stockmann C, et al. Differential activation and antagonistic function of HIF- $\{$ alpha\} isoforms in macrophages are essential for NO homeostasis. Genes Dev. 2010;24:491-501.

73. Xuan D, Han Q, Tu Q, Zhang L, Yu L, Murry D, et al. Epigenetic modulation in periodontitis: interaction of adiponectin and JMJD3-IRF4 axis in macrophages. J Cell Physiol. 2016;231:1090-6.

74. Satoh T, Takeuchi O, Vandenbon A, Yasuda K, Tanaka Y, Kumagai Y, et al. The Jmjd3-Irf4 axis regulates M2 macrophage polarization and host responses against helminth infection. Nat Immunol. 2010;11:936-44.

75. De Santa F, Totaro MG, Prosperini E, Notarbartolo S, Testa G, Natoli G. The histone H3 lysine-27 demethylase Jmjd3 links inflammation to inhibition of polycomb-mediated gene silencing. Cell. 2007;130:1083-94.

76. Krausgruber T, Blazek K, Smallie T, Alzabin S, Lockstone H, Sahgal N, et al. IRF5 promotes inflammatory macrophage polarization and TH1-TH17 responses. Nat Immunol. 2011;12:231-8.

77. Bouhlel MA, Derudas B, Rigamonti E, Dièvart R, Brozek J, Haulon S, et al. PPARgamma activation primes human monocytes into alternative M2 macrophages with anti-inflammatory properties. Cell Metab. 2007;6:137-43.

78. Huang JT, Welch JS, Ricote M, Binder CJ, Willson TM, Kelly $\mathrm{C}$, et al. Interleukin-4-dependent production of PPAR-gamma ligands in macrophages by 12/15-lipoxygenase. Nature. 1999;400:378-82.

79. Szanto A, Balint BL, Nagy ZS, Barta E, Dezso B, Pap A, et al. STAT6 transcription factor is a facilitator of the nuclear receptor PPAR $\gamma$-regulated gene expression in macrophages and dendritic cells. Immunity. 2010;33:699-712.

80. Pello OM, De Pizzol M, Mirolo M, Soucek L, Zammataro L, Amabile A, et al. Role of c-MYC in alternative activation of human macrophages and tumor-associated macrophage biology. Blood. 2012;119:411-21.

81. Andreoli V, Gehrau RC, Bocco JL. Biology of Krüppel-like factor 6 transcriptional regulator in cell life and death. IUBMB Life. 2010;62:896-905.

82. Liao X, Sharma N, Kapadia F, Zhou G, Lu Y, Hong H, et al. Krüppel-like factor 4 regulates macrophage polarization. J Clin Invest. 2011;121:2736-49.

83. Date D, Das R, Narla G, Simon DI, Jain MK, Mahabeleshwar $\mathrm{GH}$. Kruppel-like transcription factor 6 regulates inflammatory macrophage polarization. J Biol Chem. 2014;289:10318-29.

84. Martinez-Nunez RT, Louafi F, Sanchez-Elsner T. The interleukin 13 (IL-13) pathway in human macrophages is modulated by microRNA-155 via direct targeting of interleukin 13 receptor alpha1 (IL13Ralpha1). J Biol Chem. 2011;286:1786-94.
85. Cai X, Yin Y, Li N, Zhu D, Zhang J, Zhang CY, et al. Re-polarization of tumor-associated macrophages to pro-inflammatory M1 macrophages by microRNA-155. J Mol Cell Biol. 2012;4:341-3.

86. Ponomarev ED, Veremeyko T, Barteneva N, Krichevsky AM, Weiner HL. MicroRNA-124 promotes microglia quiescence and suppresses EAE by deactivating macrophages via the C/EBP$\alpha$-PU.1 pathway. Nat Med. 2011;17:64-70.

87. Zhuang G, Meng C, Guo X, Cheruku PS, Shi L, Xu H, et al. A novel regulator of macrophage activation: miR-223 in obesity-associated adipose tissue inflammation. Circulation. 2012;125:2892-903.

88. Banerjee S, Xie N, Cui H, Tan Z, Yang S, Icyuz M, et al. MicroRNA let-7c regulates macrophage polarization. J Immunol. 2013;190:6542-9.

89. Tan SY, Krasnow MA. Developmental origin of lung macrophage diversity. Development. 2016;143:1318-27.

90. Shibata Y, Berclaz PY, Chroneos ZC, Yoshida M, Whitsett JA, Trapnell BC. GM-CSF regulates alveolar macrophage differentiation and innate immunity in the lung through PU.1. Immunity. 2001;15:557-67.

91. Aguzzi A, Barres BA, Bennett ML. Microglia: scapegoat, saboteur, or something else. Science. 2013;339:156-61.

92. Duan M, Li WC, Vlahos R, Maxwell MJ, Anderson GP, Hibbs ML. Distinct macrophage subpopulations characterize acute infection and chronic inflammatory lung disease. J Immunol. 2012;189:946-55.

93. Short KR, Kroeze E, Fouchier R, Kuiken T. Pathogenesis of influenza-induced acute respiratory distress syndrome. Lancet Infect Dis. 2014;14:57-69.

94. Higgins DM, Sanchez-Campillo J, Rosas-Taraco AG, Higgins JR, Lee EJ, Orme IM, et al. Relative levels of M-CSF and GMCSF influence the specific generation of macrophage populations during infection with Mycobacterium tuberculosis. J Immunol. 2008;180:4892-900.

95. Laskin DL, Malaviya R, Laskin JD. Role of macrophages in acute lung injury and chronic fibrosis induced by pulmonary toxicants. Toxicol Sci. 2019;168:287-301.

96. Wang J, Li R, Peng Z, Zhou W, Hu B, Rao X, et al. GTS-21 reduces inflammation in acute lung injury by regulating M1 polarization and function of alveolar macrophages. Shock. 2019;51:389-400.

97. Zhuo Y, Li D, Cui L, Li C, Zhang S, Zhang Q, et al. Treatment with 3,4-dihydroxyphenylethyl alcohol glycoside ameliorates sepsis-induced ALI in mice by reducing inflammation and regulating M1 polarization. Biomed Pharmacother. 2019;116:109012.

98. Wang Y, Xu Y, Zhang P, Ruan W, Zhang L, Yuan S, et al. Smiglaside A ameliorates LPS-induced acute lung injury by modulating macrophage polarization via AMPK-PPAR $\gamma$ pathway. Biochem Pharmacol. 2018;156:385-95.

99. Bittencourt-Mernak MI, Pinheiro NM, Santana FP, Guerreiro MP, Saraiva-Romanholo BM, Grecco SS, et al. Prophylactic and therapeutic treatment with the flavonone sakuranetin ameliorates LPS-induced acute lung injury. Am J Physiol Lung Cell Mol Physiol. 2017;312:L217-L230230.

100. Pinheiro NM, Santana FP, Almeida RR, Guerreiro M, Martins MA, Caperuto LC, et al. Acute lung injury is reduced by the $\alpha 7 n A C h R$ agonist PNU-282987 through changes in the macrophage profile. FASEB J. 2017;31:320-32.

101. Dolinay T, Kaminski N, Felgendreher M, Kim HP, Reynolds P, Watkins SC, et al. Gene expression profiling of target genes in ventilator-induced lung injury. Physiol Genomics. 2006;26:68-75.

102. Johnston LK, Rims CR, Gill SE, McGuire JK, Manicone AM. Pulmonary macrophage subpopulations in the induction and resolution of acute lung injury. Am J Respir Cell Mol Biol. 2012;47:417-26. 
103. Herold S, Mayer K, Lohmeyer J. Acute lung injury: how macrophages orchestrate resolution of inflammation and tissue repair. Front Immunol. 2011;2:65.

104. Shirey KA, Pletneva LM, Puche AC, Keegan AD, Prince GA, Blanco JC, et al. Control of RSV-induced lung injury by alternatively activated macrophages is IL-4R alpha-, TLR4-, and IFN-beta-dependent. Mucosal Immunol. 2010;3:291-300.

105. Tu GW, Shi Y, Zheng YJ, Ju MJ, He HY, Ma GG, et al. Glucocorticoid attenuates acute lung injury through induction of type 2 macrophage. J Transl Med. 2017;15:181.

106. Thompson BT, Chambers RC, Liu KD. Acute respiratory distress syndrome. N Engl J Med. 2017;377:562-72.

107. Strieter RM. What differentiates normal lung repair and fibrosis? Inflammation, resolution of repair, and fibrosis. Proc Am Thorac Soc. 2008;5:305-10.

108. Lupher ML Jr, Gallatin WM. Regulation of fibrosis by the immune system. Adv Immunol. 2006;89:245-88.

109. Sinha P, Clements VK, Ostrand-Rosenberg S. Interleukin-13-regulated M2 macrophages in combination with myeloid suppressor cells block immune surveillance against metastasis. Cancer Res. 2005;65:11743-51.
110. Tsoutsou PG, Gourgoulianis KI, Petinaki E, Germenis A, Tsoutsou AG, Mpaka M, et al. Cytokine levels in the sera of patients with idiopathic pulmonary fibrosis. Respir Med. 2006;100:938-45.

111. Meneghin A, Hogaboam CM. Infectious disease, the innate immune response, and fibrosis. J Clin Invest. 2007;117:530-8.

112. D'Alessio FR, Craig JM, Singer BD, Files DC, Mock JR, Garibaldi BT, et al. Enhanced resolution of experimental ARDS through IL-4-mediated lung macrophage reprogramming. Am J Physiol Lung Cell Mol Physiol. 2016;310:L733-L746746.

113. Wakayama H, Hashimoto N, Matsushita Y, Matsubara K, Yamamoto N, Hasegawa Y, et al. Factors secreted from dental pulp stem cells show multifaceted benefits for treating acute lung injury in mice. Cytotherapy. 2015;17:1119-29.

Publisher's Note Springer Nature remains neutral with regard to jurisdictional claims in published maps and institutional affiliations. 